\title{
Preliminary Clinical Outcomes of a New Enhanced Depth of Focus Intraocular Lens
}

\author{
Nuno Campos ${ }^{1,2, *}$ \\ Tomás Loureiro (iD) ${ }^{1, *}$ \\ Sandra Rodrigues-Barros ${ }^{1,2}$ \\ Ana Rita Carreira (D) \\ Filipe Moraes id ' \\ Pedro Carreira (D) \\ Inês Machado ${ }^{1,2}$ \\ 'Ophthalmology Department, Hospital \\ Garcia de Orta, Almada, Portugal; \\ ${ }^{2}$ Ophthalmology Department, Hospital \\ CUF, Lisboa, Portugal \\ *These authors contributed equally to \\ this work
}

\begin{abstract}
Purpose: To assess the preliminary clinical outcomes and patient satisfaction of the new enhanced depth of focus (EDOF) LuxSmart ${ }^{\mathrm{TM}}$ intraocular lens IOL and to compare with a conventional monofocal IOL $\left(\right.$ Akreos $\left.^{\mathrm{TM}}\right)$ in patients who had undergone bilateral cataract surgery.

Methods: Twelve patients underwent bilateral LuxSmart IOL implantation, and twelve underwent bilateral Akreos IOL implantation. Best-corrected distance (CDVA) and uncorrected distance visual acuity (UDVA), uncorrected intermediate visual acuity (UIVA) at $66 \mathrm{~cm}$, uncorrected near visual acuity (UNVA) at $40 \mathrm{~cm}$ and defocus curve were assessed. Patients-reported visual function was inquired by Catquest-9SF. The presence of photic phenomena was evaluated. A p-value lower than 0.05 was considered for statistical significance.

Results: The mean IOL power was $+21.90 \mathrm{D}$ in LuxSmart group and $+22.30 \mathrm{D}$ in Akreos. Monocular UDVA $(p=0.32)$ and CDVA $(p=0.52)$ did not differ between groups. The average binocular UIVA $(0.18 \pm 0.12 \log$ MAR vs. $0.30 \pm 0.13 \operatorname{logMAR}, \mathrm{p}<0.001)$ and UNVA $(0.38 \pm 0.14 \log$ MAR vs. $0.44 \pm 0.17 \log$ MAR, $\mathrm{p}=0.02)$ were higher in LuxSmart IOL group. No patients reported disabling photic phenomena in either group.

Conclusion: This study shows that new LuxSmart EDOF IOL achieved higher performance for intermediate and near vision compared with a conventional monofocal IOL, without increasing the risk of dysphotopsias. LuxSmart may be an attractive and safe option for patients who desire spectacle independence for distance and intermediate vision after cataract surgery.
\end{abstract}

Keywords: cataract, presbyopia, IOL, EDOF, LuxSmart, enhanced depth of focus, intraocular lens

\section{Introduction}

Cataract surgery has evolved considerably in the last decades. Surgical technique improvements, higher intraocular lens (IOL) calculation formulas accuracy and development of many advanced IOLs increased refractive issues associated with cataract surgery. Nowadays, patients have higher expectations and some desire spectacle independence for both distance and near. The increasing use of technology raised concerns regarding intermediate vision. ${ }^{1}$ Multifocal IOLs were designed to simultaneously provide good distance and near visions but usually provide insufficient intermediate vision. ${ }^{2}$ Furthermore, they can be associated with visually significant photic phenomena which could be very unpleasant and require very meticulous patient selection. ${ }^{3}$ As such, monofocal IOLs are still the most implanted IOLs in cataract surgery and there is gap to fill with an IOL that offers a good balance between uncorrected distance and intermediate vision and photic phenomena.

To overcome the issues of multifocal IOLs, there is a growing interest toward new technologies that may improve the intermediate performance of monofocal
Correspondence: Tomás Loureiro Email Loureiro.tomas@gmail.com 
IOLs. LuxSmart ${ }^{\mathrm{TM}}$ IOL (Bausch \& Lomb GmbH, Berlin, Germany) is a newer-generation $6.0 \mathrm{~mm}$ single piece, acrylic hydrophobic and aspheric IOL. Its design creates a small central zone which combines 4 th and 6th orders of spherical aberration of opposite signs to increase the subjective depth of focus and further improve intermediate vision while maintaining good performance at distance.

This study aims to present a case series to evaluate the preliminary visual outcomes and vision-related quality of life of the new enhanced depth of focus (EDOF) IOL LuxSmart - and to compare with a conventional monofocal IOL - Akreos ${ }^{\mathrm{TM}}$ - in patients who had undergone bilateral cataract surgery.

\section{Methods}

This was a consecutive case series single-center study that included patients aged between 65 and 80 years who had undergone bilateral consecutive cataract surgery in the period comprised between April and May of 2021 in Ophthalmology Department of Hospital Garcia de Orta (Almada, Portugal). Patients were randomly selected to receive either an EDOF IOL, LuxSmart (Bausch \& Lomb GmbH, Berlin, Germany) or a conventional monofocal IOL, Akreos (Bausch \& Lomb GmbH, Berlin, Germany). Inclusion criteria were clinical indication for cataract surgery, corneal regular astigmatism no more than 0.75 diopters (D), photopic pupil diameter higher than $2 \mathrm{~mm}$ and axial length between 22.5 and $24.99 \mathrm{~mm}$. Exclusion criteria included diabetes mellitus, previous ophthalmological surgery, corneal pathology, and retinal and optic nerve diseases. The study was conducted by the principles of the Declaration of Helsinki and all participants were informed about the purpose. Signed consent was obtained from all patients and approval was obtained from the institutional Research Committee of Hospital Garcia de Orta (Almada, Portugal).

Preoperative evaluation included anterior segment biomicroscopy, Goldmann applanation tonometry and indirect fundoscopy. Specular microscopy was performed with Topcon Specular Microscope SP-1P (Tokyo, Japan). Biometry was performed using IOL Master 700 (Carl Zeiss, Oberkochen, Germany) and IOL power was calculated targeting emmetropia using the Barrett Universal II Formula and the A-constant was 118.6. Cataract surgeries were performed by four surgeons via a $2.4 \mathrm{~mm}$ clear corneal incision. For astigmatism management, this incision was performed in the steepest meridian (measured by Scheimpflug tomography) in eyes having a corneal astigmatism greater than 0.50 D. A $5.5 \mathrm{~mm}$ capsulorhexis was performed, the cataract was removed using phacoemulsification technique and the IOL was implanted in the capsular bag. Patients were medicated with a tapered dose of topical steroids and with topical non-steroid anti-inflammatory for one month and topical antibiotic for two weeks.

Patients were evaluated at postoperative day one, week one, month one and three months after the second eye surgery where an exhaustive evaluation was performed. The last evaluation was performed by the same surgeon in every patient. The primary outcomes measured were monocular and binocular distance (at $6 \mathrm{~m}$ ), intermediate (at $66 \mathrm{~cm}$ ) and near (at $40 \mathrm{~cm}$ ) visual acuity (presented in Snellen scale and $\log$ MAR). The manifest refraction, monocular and binocular corrected (CDVA) and uncorrected (UDVA) visual acuity, uncorrected intermediate visual acuity (UIVA) and uncorrected near visual acuity (UNVA) were tested. Monocular distance-corrected defocus curve was also assessed from $-3.50 \mathrm{D}$ to $+1.50 \mathrm{D}$ in $0.5 \mathrm{D}$ increments under photopic lighting conditions.

Catquest-9SF questionnaire was given to the patients to evaluate patients-reported unaided visual function as related to day-to-day task. The presence of photic phenomena (halo, glare and starburst) was also assessed by showing standard photographs to the patients. Emphasis to nocturne driving was given.

Statistical analysis was made by $\operatorname{SPSS}^{\mathrm{TM}}$ software version 23.0. Qualitative variables are presented as number and percentage. Data were confirmed to be normally distributed by Shapiro-Wilk test. Two tailed Student's $\mathrm{T}$ tests were applied to compare means by groups. The percentages of each answer on the Catquest-9SF questionnaire were calculated and compared between groups. Statistical significance was defined as $<0.05$.

\section{Results}

This study comprised a total of 48 eyes of 24 patients. Twelve patients $(50 \%$ males and $50 \%$ females $)$ were included in each IOL group. The mean age did not differ between groups ( $68 \pm 6.7$, range $64-76$ vs. $71 \pm 5.4$, range 67-79 $\mathrm{p}=0.42$ ). Demographic and biometric data are presented in Table 1 . The mean preoperative spherical equivalent did not differ between groups $(\mathrm{p}=0.52)$. No patients were omitted, and no one was lost to follow-up.

The clinical outcomes at three months after surgery and respective comparisons between groups are presented in Table 2. The mean LuxSmart power was +21.90D and $+22.30 \mathrm{D}$ in Akreos $(\mathrm{p}=0.33)$. The final spherical 
Table I Demographic and Biometric Data

\begin{tabular}{|l|c|c|c|}
\hline & LuxSmart & Akreos & p-value \\
\hline Number of Patient/Eyes (n) & $12 / 24$ & $12 / 24$ & - \\
\hline Female (\%) & $50 \%$ & $50 \%$ & 0.42 \\
\hline Age (y) \pm SD & $68 \pm 6.7$ & $71 \pm 5.4$ & 0.31 \\
\hline Axial length (mm) \pm SD & $23.3 \pm 0.7$ & $22.8 \pm 0.8$ & 0.42 \\
\hline KI (D) \pm SD & $43.6 \pm 1.1$ & $44.1 \pm 0.9$ & 0.52 \\
\hline K2 (D) \pm SD & $44.3 \pm 1.3$ & $44.5 \pm 1.2$ & 0.33 \\
\hline IOL power (D) \pm SD & $21.9 \pm 1.3$ & $22.3 \pm 1.1$ & \\
\hline
\end{tabular}

Abbreviations: SD, Standard deviation; KI, Flat corneal meridian; K2, Steep corneal meridian; D, Diopters; IOL, Intraocular lens.

Table 2 Clinical Outcomes

\begin{tabular}{|l|c|c|c|c|}
\hline Clinical Outcomes (3 Months) & LuxSmart & Akreos & p-value \\
\hline \multirow{3}{*}{ Binocular } & CDVA & $20 / 20(-0.08 \pm 0.04)$ & $20 / 20(-0.02 \pm 0.07)$ & 0.52 \\
\cline { 2 - 5 } & UDVA & $20 / 20(0.02 \pm 0.11)$ & $20 / 20(0.01 \pm 0.10)$ & 0.71 \\
\cline { 2 - 5 } & UIVA & $20 / 32(0.18 \pm 0.12)$ & $20 / 40(0.30 \pm 0.13)$ & $<0.001$ \\
\cline { 2 - 5 } & UNVA & $20 / 32(0.38 \pm 0.14)$ & $20 / 40(0.44 \pm 0.17)$ & $\mathbf{0 . 0 2}$ \\
\hline \multirow{3}{*}{ Monocular } & CDVA & $20 / 20(-0.04 \pm 0.01)$ & $20 / 20(-0.01 \pm 0.03)$ & 0.66 \\
\cline { 2 - 6 } & UDVA & $20 / 22(0.10 \pm 0.11)$ & $20 / 25(0.01 \pm 0.15)$ & 0.62 \\
\hline Snellen (logMAR) & UIVA & $20 / 32(0.21 \pm 0.08)$ & $20 / 40(0.36 \pm 0.13)$ & $<0.001$ \\
\hline
\end{tabular}

Note: Significant statistic difference is highlighted by $\mathrm{P}$-values in bold.

Abbreviations: logMAR, Logarithm of minimum angle of resolution; D, Diopters.

equivalent was $-0.08 \pm 0.6 \mathrm{D}$ in LuxSmart and $-0.12 \pm 0.4$ $\mathrm{D}$ in Akreos group ( $\mathrm{p}=0.37$ ). $91.7 \%$ of eyes in LuxSmart group and $87.5 \%$ in Akreos group were within 0.5D of emmetropic target. Every patient achieved binocular and monocular CDVA of at least 20/20, with no differences between groups $(\mathrm{p}=0.52$ and $\mathrm{p}=0.66$, respectively). Intermediate visual acuities, both binocular and monocular, were higher in LuxSmart group $(\mathrm{p}<0.001)$ with all patients achieving UIVA better than 20/40. The mean binocular and monocular UIVA in LuxSmart group were $20 / 32(0.18 \pm 0.12 \operatorname{logMAR})$ and $20 / 32(0.21 \pm 0.08$ $\log$ MAR), respectively. Uncorrected near visual acuity was higher in LuxSmart group (20/32 or $0.38 \pm 0.14$ $\log$ MAR vs. $20 / 40$ or $0.44 \pm 0.17 \log$ MAR, $\mathrm{p}=0.02$ ).

The defocus curves for both IOLs is presented in Figure 1. LuxSmart provided a sustained visual acuity of $0.3 \log$ MAR or better between $+1.00 \mathrm{D}$ and $-2.00 \mathrm{D}$.
Akreos provided visual acuity of $0.3 \log$ MAR or better between +1.00 and $-1.00 \mathrm{D}$, from which dropped abruptly.

The prevalence of photic phenomena is presented in Table 3. Most patients reported no photic phenomena, both in LuxSmart (66.6\%) and in Akreos (66.7\%) groups. None reported starburst. None had disabling halos or glare and there were no differences between groups regarding halos and glare ( $\mathrm{p}=0.89$ and $\mathrm{p}=0.66$, respectively).

The results of the Catquest-9SF questionnaire are presented in Table 4. Most patients in LuxSmart group achieved higher degrees of satisfaction for daily life activities, especially in reading newspapers, reading prices while shopping and using computer. Regarding activities which require distance vision, patients reported same facilities on both groups. The patients were more satisfied with their visual acuity in LuxSmart group: none were dissatisfied and $83.3 \%$ were very satisfied. 


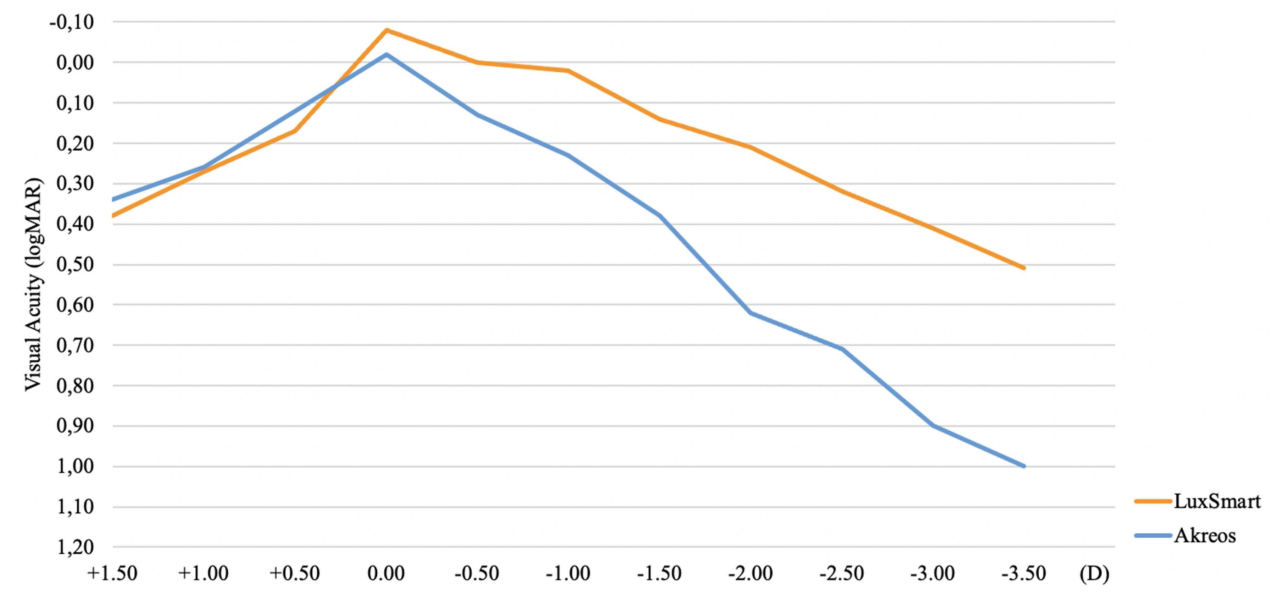

Figure I Monocular distance-corrected defocus curve three months after binocular implantation of IOL. Abbreviations: IOL, Intraocular lens; logMAR, Logarithm of minimum angle of resolution; D, Diopters.

\section{Discussion}

The present study shows that new LuxSmart EDOF IOL achieves better performance for intermediate vision than a conventional monofocal IOL, without compromising distance vision. Furthermore, LuxSmart IOL did not increase risk of photic phenomena.

There are raising concerns regarding presbyopia correction at the time of cataract surgery. ${ }^{4}$ From the available options, it is important to clarify their differences to better fit each one according to patient needs and expectations. Monovision with monofocal IOLs, multifocal IOLs and EDOF IOLs are the most accepted strategies but provide different outcomes regarding intermediate and near vision. ${ }^{5,6}$ Monovision have the highest rate of spectacle dependence. ${ }^{7}$ Multifocal IOLs provide good near vision but have the highest rates of halos and glare and tend to sacrifice contrast sensitivity. ${ }^{8,9}$ EDOF IOLs tend to provide excellent intermediate vision without compromising distance vision but near vision is often insufficient for spectacle independence. ${ }^{2}$
Recent developments in cataract surgery, in particularly regarding IOL formulas accuracy, enabled spectacle independence for distance vision. There are no clear advantages regarding distance vision between IOLs available. $^{2}$ In this article, in both LuxSmart and Akreos groups, binocular uncorrected visual acuity was excellent. However, this also result from our tight inclusion criteria, such as lower corneal astigmatism and narrow axial length interval included which resulted in predictable low residual refractive errors. However, early refractive results from the LuxSmart IOL showed a myopic tendency, so A constant was changed and found to be more accurate.

Intermediate vision is a hot topic in ophthalmology as people increasingly use technological devices. ${ }^{10}$ Our study showed advantage of LuxSmart regarding uncorrected and distance-corrected intermediate visual acuity as shown by the smooth transition between distance and intermediate vision in defocus curve. EDOF IOLs have previously proved to achieve better intermediate vision than monofocal and

Table 3 Incidence of Photic Phenomena

\begin{tabular}{|c|c|c|c|c|c|c|}
\hline \multirow{2}{*}{$\begin{array}{l}\text { Photic } \\
\text { Phenomena }\end{array}$} & \multicolumn{2}{|c|}{ Halos } & \multirow[b]{2}{*}{ p-value } & \multicolumn{2}{|c|}{ Glare } & \multirow[b]{2}{*}{ p-value } \\
\hline & LuxSmart & Akreos & & LuxSmart & Akreos & \\
\hline Never & $8(66.7 \%)$ & 8 (66.7\%) & & $8(66.7 \%)$ & $8(66.7 \%)$ & 0.66 \\
\hline Rarely & 2 (16.7\%) & 2 (16.7\%) & & $3(25 \%)$ & $4(33.3 \%)$ & \\
\hline Sometimes & I (8.3\%) & I (8.3\%) & 0.89 & I (8.3\%) & $0(0 \%)$ & \\
\hline Often & I (8.3\%) & I (8.3\%) & & $0(0 \%)$ & $0(0 \%)$ & \\
\hline Always & $0(0 \%)$ & $0(0 \%)$ & & $0(0 \%)$ & $0(0 \%)$ & \\
\hline
\end{tabular}


Table 4 Results of Catquest 9-SF (Uncorrected Vision)

\begin{tabular}{|c|c|c|c|c|}
\hline & & LuxSmart & Akreos & p-value \\
\hline & & $\mathrm{n}(\%)$ & $\mathrm{n}(\%)$ & \\
\hline \multirow[t]{5}{*}{ Reading newspaper print } & Yes, very great difficulty & 2 & 5 & \\
\hline & Yes, great dificulty & 2 & 4 & \\
\hline & Yes, some difficulty & 2 & 2 & 0.01 \\
\hline & No, no difficulty & 6 & I & \\
\hline & Cannot decide & 0 & 0 & \\
\hline \multirow[t]{5}{*}{ Recognizing the faces of people } & Yes, very great difficulty & 0 & 0 & \\
\hline & Yes, great dificulty & 0 & 0 & \\
\hline & Yes, some difficulty & 1 & I & 0.52 \\
\hline & No, no difficulty & 11 & 11 & \\
\hline & Cannot decide & 0 & 0 & \\
\hline \multirow{5}{*}{$\begin{array}{l}\text { Reading the prices of goods while } \\
\text { shopping }\end{array}$} & Yes, very great difficulty & 0 & 9 & \\
\hline & Yes, great dificulty & 0 & 2 & \\
\hline & Yes, some difficulty & 2 & I & 0.001 \\
\hline & No, no difficulty & 10 & 0 & \\
\hline & Cannot decide & 0 & 0 & \\
\hline \multirow[t]{5}{*}{ Seeing to walk on uneven ground } & Yes, very great difficulty & 0 & 0 & \\
\hline & Yes, great dificulty & 0 & 0 & \\
\hline & Yes, some difficulty & I & I & 0.66 \\
\hline & No, no difficulty & II & 11 & \\
\hline & Cannot decide & 0 & 0 & \\
\hline \multirow[t]{5}{*}{ Reading television text } & Yes, very great difficulty & 0 & 0 & \\
\hline & Yes, great dificulty & I & 2 & \\
\hline & Yes, some difficulty & I & I & 0.52 \\
\hline & No, no difficulty & 10 & 9 & \\
\hline & Cannot decide & 0 & 0 & \\
\hline \multirow[t]{5}{*}{ Seeing to use the computer } & Yes, very great difficulty & 0 & 4 & \\
\hline & Yes, great dificulty & 1 & 4 & \\
\hline & Yes, some difficulty & 3 & 2 & 0.001 \\
\hline & No, no difficulty & 8 & 2 & \\
\hline & Cannot decide & 0 & 0 & \\
\hline
\end{tabular}

(Continued) 
Table 4 (Continued).

\begin{tabular}{|c|c|c|c|c|}
\hline & & LuxSmart & Akreos & p-value \\
\hline \multirow{5}{*}{$\begin{array}{l}\text { Difficulties in performing everyday } \\
\text { tasks }\end{array}$} & Yes, very great difficulty & 0 & 0 & \\
\hline & Yes, great dificulty & 0 & 0 & \\
\hline & Yes, some difficulty & 5 & 10 & 0.07 \\
\hline & No, no difficulty & 7 & 2 & \\
\hline & Cannot decide & 0 & 0 & \\
\hline \multirow{4}{*}{$\begin{array}{l}\text { Satisfied or dissatisfied with your } \\
\text { present vision }\end{array}$} & Very satisfied & 10 & 6 & \\
\hline & Rather satisfied & 2 & 5 & \\
\hline & Rather dissatisfied & 0 & I & 0.04 \\
\hline & Very dissatisfied & 0 & 0 & \\
\hline
\end{tabular}

Note: Significant statistic difference is highlighted by $\mathrm{p}$-values in bold.

trifocal IOLs. ${ }^{2,11}$ Cochener and Sachdev et al reported a mean binocular UIVA of $0.12 \log$ MAR and $0.01 \log$ MAR with Tecnis Symphony IOL, respectively. ${ }^{12,13}$ Differently from monofocal IOLs, the center of LuxSmart EDOF IOL contains a refractive optic that combines fourth and sixthorder spherical aberration of opposite signs to increase the depth of focus.

Despite EDOF IOLs were designed to optimize independence for distance and intermediate vision, preliminary LuxSmart IOL outcomes showed surprisingly satisfactory results for near vision. In this article, LuxSmart group achieved better near visual acuity than Akreos group and patients reported facility to read newspapers without spectacles. The wide range of defocus curve is beyond these results. However, $50 \%$ of patients in LuxSmart group and all in Akreos group reported spectacle depended for near vision, specially to read small letter which is in line with EDOF IOLs previous studies. ${ }^{2,12}$ Here, aiming mini monovision could be a promising strategy to enhance spectacle independence.

Trifocal IOLs usually provide spectacle independence, for both distance and near vision. However, trifocal IOLs are associated with a considerable rate of dysphotopsias and patient dissatisfaction. ${ }^{8,14-16}$ Jacobi et al reported an incidence of $25 \%$ and $29 \%$ of halos and glare with multifocal IOLs at the 6 th postoperative month, respectively. ${ }^{17}$ EDOF IOLs have a single-elongated focal point to enhance the depth of focus instead of having two or three discrete points. As such, EDOF IOLs seem to be more friendly regarding photic phenomena. Moreover, LuxSmart is one of few EDOL IOLs that do not depend on the diffractive principle and therefore should not cause more halos and glare than monofocal IOLs. In this article, the rate of dysphotopsias was quite low and there were no differences between monofocal and EDOF IOLs. Furthermore, we believe this rate would be lower if the patients were evaluated later because neuroadaptation could last six months.

More than providing spectacle independence, refractive cataract surgery aims to improve the quality of life. Patients' satisfaction is difficult to evaluate and depends on many variables. Catquest 9-SF is a validated questionnaire which was designed to evaluate the performance of uncorrected visual acuity and associated patient satisfaction. ${ }^{18}$ Overall satisfaction was good in both groups. However, it was higher in LuxSmart group. As patients of LuxSmart group reported less difficulties in reading newspapers, reading prices at supermarkets and using computers, we postulate that better intermediate vision is associated with higher quality of life.

Limitations of our study are the small sample and the short follow-up period. Despite these limitations, we believe that these preliminary results show that LuxSmart IOL provides better intermediate vision than a monofocal IOL without compromising distance vision. The low incidence of dysphotpsias, makes LuxSmart an attractive option for patients who desire spectacle independence for distance and working intermediate vision after cataract surgery.

\section{Conclusion}

The preliminary results show that new LuxSmart EDOF IOL achieved higher performance for uncorrected 
intermediate and near vision compared with a conventional monofocal IOL, without increasing the risk of photic phenomena. LuxSmart may be an attractive and safe option for patients who desire spectacle independence for distance and intermediate vision after cataract surgery.

\section{Funding}

No financial disclosures to declare.

\section{Disclosure}

The authors have no conflicts of interest to declare.

\section{References}

1. de Asís Bartol-Puyal F, Talavero P, Giménez G, et al. Reading and quality of life differences between Tecnis ZCB00 monofocal and Tecnis ZMB00 multifocal intraocular lenses. Eur $J$ Ophthalmol. 2017;27(4):443-453. doi:10.5301/ejo.5000925

2. Böhm M, Petermann K, Hemkeppler E, Kohnen T. Defocus curves of 4 presbyopia-correcting IOL designs: diffractive panfocal, diffractive trifocal, segmental refractive, and extended-depth-of-focus. J Cataract Refract Surg. 2019;45(11):1625-1636. doi:10.1016/j.jcrs.2019.07.014

3. Häring G, Dick BH, Krummenauer F, Weissmantel U, Kröncke W. Subjective photic phenomena with refractive multifocal and monofocal intraocular lenses: results of a multicenter questionnaire. $J$ Cataract Refract Surg. 2001;27(2):245-249. doi:10.1016/S08863350(00)00540-X

4. Shafer BM, Greenwood M. Presbyopia correction at the time of cataract surgery. Curr Ophthalmol Rep. 2020;8(3):79-87. doi:10.1007/s40135-020-00236-y

5. Greenwood M, Bafna S, Thompson V. Surgical correction of presbyopia: lenticular, corneal, and scleral approaches. Int Ophthalmol Clin. 2016;56(3):149-166. doi:10.1097/IIO.0000000000000124

6. Akella SS, Juthani VV. Extended depth of focus intraocular lenses for presbyopia. Curr Opin Ophthalmol. 2018;29(4):318-322. doi:10.1097/ ICU.0000000000000490

7. Boerner CF, Thrasher BH. Results of monovision correction in bilateral pseudophakes. J Am Intraocul Implant Soc. 1984;10(1):49-50. doi:10.1016/S0146-2776(84)80077-4
8. Tchah H, Nam K, Yoo A. Predictive factors for photic phenomena after refractive, rotationally asymmetric, multifocal intraocular lens implantation. Int J Ophthalmol. 2017;10(2):241-245. doi:10.18240/ ijo.2017.02.10

9. Kelava L, Barić H, Bušić M, Čima I, Trkulja V. Monovision versus multifocality for presbyopia: systematic review and meta-analysis of randomized controlled trials. Adv Ther. 2017;34(8):1815-1839. doi:10.1007/s12325-017-0579-7

10. Grzybowski A, Kanclerz P. Recent developments in cataract surgery. In: Grzybowski A, editor. Current Concepts in Ophthalmology. Cham: Springer International Publishing; 2020:55-97. doi:10.1007/ 978-3-030-25389-9 3

11. Kanclerz P, Toto F, Grzybowski A, Alio JL. Extended depth-of-field intraocular lenses: an update. Asia Pac J Ophthalmol. 2020;9 (3):194-202. doi:10.1097/APO.0000000000000296

12. Cochener B; Group for the CS. Clinical outcomes of a new extended range of vision intraocular lens: International Multicenter Concerto Study. J Cataract Refract Surg. 2016;42(9):1268-1275. doi:10.1016/ j.jcrs.2016.06.033

13. Sachdev GS, Ramamurthy S, Sharma U, Dandapani R. Visual outcomes of patients bilaterally implanted with the extended range of vision intraocular lens: a prospective study. Indian $J$ Ophthalmol. 2018;66(3):407.

14. Buckhurst PJ, Naroo SA, Davies LN, Shah S, Drew T, Wolffsohn JS. Assessment of dysphotopsia in pseudophakic subjects with multifocal intraocular lenses. BMJ Open Ophthalmol. 2017;1(1):e000664. doi:10.1136/bmjophth-2016-000064

15. de Vries NE, Webers CAB, Touwslager WRH, et al. Dissatisfaction after implantation of multifocal intraocular lenses. $J$ Cataract Refract Surg. 2011;37(5):859-865. doi:10.1016/j.jcrs.2010.11.032

16. Woodward MA, Randleman BJ, Stulting DR. Dissatisfaction after multifocal intraocular lens implantation. J Cataract Refract Surg. 2009;35(6):992-997. doi:10.1016/j.jcrs.2009.01.031

17. Jacobi FK, Kammann J, Jacobi KW, Großkopf U, Walden K. Bilateral implantation of asymmetrical diffractive multifocal intraocular lenses. Arch Ophthalmol. 1999;117(1):17-23. doi:10.1001/ archopht.117.1.17

18. Lundström M, Pesudovs K. Catquest-9SF patient outcomes questionnaire: nine-item short-form Rasch-scaled revision of the Catquest questionnaire. J Cataract Refract Surg. 2009;35(3):504-513. doi:10.1016/j.jcrs.2008.11.038
Clinical Ophthalmology

\section{Publish your work in this journal}

Clinical Ophthalmology is an international, peer-reviewed journal covering all subspecialties within ophthalmology. Key topics include: Optometry; Visual science; Pharmacology and drug therapy in eye diseases; Basic Sciences; Primary and Secondary eye care; Patient Safety and Quality of Care Improvements. This journal is indexed on PubMed

\section{Dovepress}

Central and CAS, and is the official journal of The Society of Clinical Ophthalmology (SCO). The manuscript management system is completely online and includes a very quick and fair peer-review system, which is all easy to use. Visit http://www.dovepress.com/ testimonials.php to read real quotes from published authors. 